[8] A. Cohen and U. Shaked, "Linear discrete-time $H_{\infty}$ optimal tracking with preview," IEEE Trans. Automat. Contr., vol. 42, pp. 270-276, 1997.

[9] T. Basar and P. Bernhard, $H_{\infty}$-Optimal Control and Related Minimax Design Problems: A Dynamic Game Approach. Boston, MA: Birkhauser, 1991.

[10] T. Basar, "A dynamic games approach to controller design: Disturbance rejection in discrete-time," IEEE Trans. Automat. Contr., vol. 36, pp. 936-952, 1991.

[11] I. Yaesh and U. Shaked, "Minimum $H_{\infty}$ norm regulation of linear discrete-time systems and its relation to linear quadratic discrete games," IEEE Trans. Automat. Contr., vol. 35, pp. 1061-1064, 1990.

[12] K. B. Kim, "A Study on Stability and Tracking Form of Intervalwise $H_{\infty}$ Control," M.S. Thesis, Dep. Control and Instrumentation, Seoul National Univ., Seoul, Korea, Feb. 1995.

[13] S. Bittanti, Deterministic and Stochastic Linear Periodic Systems. Berlin, Germany: Springer-Verlag, 1986.

\section{Localization Based Switching Adaptive Control for Time-Varying Discrete-Time Systems}

Peter V. Zhivoglyadov, Richard H. Middleton, and Minyue Fu

\begin{abstract}
In this paper a new systematic switching control approach to adaptive stabilization of linear time-varying (LTV) discrete-time systems is presented. A feature of the localization-based method is its high model falsification capability, which in the case of LTI systems is manifested as the rapid convergence of the switching controller. We believe that the proposed method may help pave the way for design of practical adaptive switching controllers applicable to a wide range of linear time-invariant and timevarying systems.
\end{abstract}

Index Terms-Adaptive control, discrete-time systems, switching control.

\section{INTRODUCTION}

It is well known [4] that classical adaptive algorithms prior to 1980 were all based on the following set of standard assumptions or variations of them: known plant order; minimum phase; known sign of high frequency gain; the plant is LTI and noise free. A number of attempts have been made since 1980 to relax the assumptions above. A major breakthrough occurred in the mid 1980's [2], [4], [8] for adaptive control of LTV plants with sufficiently small mean parameter variations.

In a separate research line, a number of switching control algorithms have been proposed recently by several authors [1], [3], [5], thus significantly weakening the assumptions above. Research in this direction originated from the pioneering works by Nussbaum [7] and Martensson [3]. A major drawback of conventional switching control based on some mechanism of an exhaustive search [1], [3] is that the search may converge very slowly, resulting in excessive transients which render the system "unstable" in a practical sense. Supervisory control for adaptive set-point tracking is proposed in a number of papers [5], [6] to improve the transient response. The main idea of the supervisory control schemes is to orchestrate the process of switching into feedback controllers from a precomputed finite (continuum) set of fixed controllers based on certain online estimation. This represents a significant depar-

Manuscript received February 11, 1998; revised June 14, 1999. Recommended by Associate Editor, A. Rantzer.

The authors are with the Department of Electrical and Computer Engineering, The University of Newcastle, N.S.W. 2308, Australia (e-mail: eepeter@ee.newcastle.edu.au; rick@ee.newcastle.edu.au; eemf@ee.newcastle.edu.au).

Publisher Item Identifier S 0018-9286(00)04100-3. ture from traditional estimator-based tuning algorithms which usually employ recursive or dynamic parameter tuning schemes. However, several issues still remain unresolved. These include the controller being nonconvergent and the proofs of stability being too complicated.

Our primary objective is to design an adaptive switching controller applicable to a wide range of LTI and LTV systems which is free from the drawbacks mentioned above. To this end, we present and analyze a new systematic approach to switching adaptive control. This approach is based on a localization method, which incorporates simultaneous falsification of a large number of models in the switching scheme. The potential advantages of localization based switching control include finite convergence for switching, simplicity of the analysis, and applicability to both LTI and LTV plants.

\section{Problem Statement}

We consider a general class of LTV discrete-time plants in the following form:

$$
D\left(t, z^{-1}\right) y(t)=N\left(t, z^{-1}\right) u(t)+\xi(t-1)+\eta(t-1)
$$

where $u(t)$ is the input, $y(t)$ is the output, $\xi(t)$ is the exogenous disturbance, $\eta(t)$ represents the unmodeled dynamics, $N\left(t, z^{-1}\right)$ and $D\left(t, z^{-1}\right)$ are polynomials in $z^{-1}$, and $z^{-1}$ is the unit delay operator. We will denote by $\theta(t)$ the vector of unknown time-varying parameters, i.e., $\theta(t)=\left(n_{n}(t), \cdots, n_{2}(t),-d_{n}(t), \cdots,-d_{1}(t), n_{1}(t)\right)^{T}$ composed of the coefficients of $N\left(t, z^{-1}\right)$ and $D\left(t, z^{-1}\right)$. Throughout the paper, we will use the following nonminimal state space description of the plant (1):

$$
x(t+1)=A(\theta(t)) x(t)+B(\theta(t)) u(t)+E(\xi(t)+\eta(t))
$$

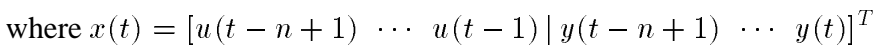
and the matrix $A(\theta(t))$ and the vectors $B(\theta(t))$ and $E$ are constructed in a standard way. We also define the regressor vector as $\phi(t)=[x(t) \mid u(t)]^{T}$. Then, (1) can be rewritten as

$$
y(t)=\phi^{T}(t-1) \theta(t-1)+\xi(t-1)+\eta(t-1) .
$$

The following assumptions [4] are used throughout the paper.

A1) The order $n$ of the nominal plant (excluding the unmodeled dynamics) is known.

A2) There exists a known compact set $\Omega \in R^{2 n}$ such that $\theta(t) \in$ $\Omega$ for all $t \in N$.

A3) The plant (1) with frozen parameters and zero unmodeled dynamics (i.e., $\eta(t) \equiv 0$ ) is stabilizable over $\Omega$.

A4) The exogenous disturbance $\xi$ is uniformly bounded, that is, $\sup _{t \geq t_{0}}|\xi(t)| \leq \bar{\xi}$ for some known constant $\bar{\xi}$.

A5) The unmodeled dynamics is arbitrary subject to $|\eta(t)| \leq \bar{\eta}(t)=\epsilon \sup _{0<k<t} \sigma^{t-k}\|x(k)\|$ for some constants $\epsilon>0$ and $0 \leq \sigma<1$ which represent the "size" and "decay rate" of the unmodeled dynamics, respectively.

A6) The uncertain parameters are allowed to have two types of time variations: i) slow parameter drift described by $\| \theta(t)-$ $\theta(t-1) \| \leq \alpha, \forall t>t_{0}$ for some constant $\alpha>0$, and ii) infrequent large jumps constrained by $\sum_{i=t}^{t+\tau N} s_{i} \leq \tau$ for all $t \geq 0$, where $\tau>0$ and $N>0$ are constants with $1 / N$ representing the "frequency" of large jumps, and $s_{i}=0$ if $\|\theta(i)-\theta(i-1)\| \leq \alpha$, and $s_{i}=1$ otherwise.

We note that Assumption A4) can be completely removed at the expense of a slightly more complicated controller (see [9] for details). 
The switching controller to be designed is of the following form: $u(t)=K_{i(t)} x(t)$ where $K_{i(t)}$ is the controller gain applied at time $t$, and $i(t)$ is the switching index taking value in a finite index set $I$. The objective of the control design is to determine the set of control gains $K_{I}=\left\{K_{i}, i \in I\right\}$ and an online switching algorithm for $i(t)$ so that the closed-loop system will be "stable" in some sense.

Definition 2.1: System (1) satisfying A1)-A6) is said to be globally $\bar{\xi}$-exponentially stabilized by the switching controller if there exist constants $M_{1}>0,0<\rho<1$, and a function $M_{2}(\cdot): \boldsymbol{R}_{+} \rightarrow \boldsymbol{R}_{+}$ with $M_{2}(0)=0$ such that $\|x(t)\| \leq M_{1} \rho^{\left(t-t_{0}\right)}\left\|x\left(t_{0}\right)\right\|+M_{2}(\bar{\xi})$ holds for all $t_{0} \geq 0, x\left(t_{0}\right), \bar{\xi} \geq 0$, and $\xi(\cdot)$ and $\eta(\cdot)$ satisfying A4) and A5), respectively.

\section{LOCALIZATION TECHNIQUE FOR LTI PLANTS}

The localization technique being the key element in the proposed method implies appropriate decomposition of the uncertainty set $\Omega$ and an effective on-line mechanism of discarding incorrect controllers. To define decomposition of $\Omega$ we use the notion of quadratic stability.

Definition 3.1: A set of matrices $\{A(\theta): \theta \in \Omega\}$ is called quadratically stable if there exist symmetric positive-definite matrices $H, Q$ such that $A^{T}(\theta) H A(\theta)-H \leq-Q, \forall \theta \in \Omega$. Now we decompose the parameter set $\Omega$ to obtain a finite cover $\left\{\Omega_{i}\right\}_{i=1}^{L}$ which satisfies the following conditions.

C1) $\Omega_{i} \subset \Omega, \Omega_{i} \neq \emptyset, i=1, \cdots, L$.

C2) $\bigcup_{i=1}^{L} \Omega_{i}=\Omega$.

C3) For each $i=1, \cdots, L, \exists \theta_{i} \in \Omega_{i}$ ("center"), $r_{i}>0$ ("raduis"), $K_{i}$ (control gain), $q>0$ (scalar parameter) and symmetric positive definite matrices $H_{i}$ and $Q_{i}$ such that $\left(A(\theta)+B(\theta) K_{i}\right)^{T} H_{i}\left(A(\theta)+B(\theta) K_{i}\right)-H_{i} \leq-Q_{i}$, $\forall\left\|\theta-\theta_{i}\right\| \leq r_{i}+q, i=1, \cdots, L$.

Conditions $\mathrm{C} 1$ ) and $\mathrm{C} 2$ ) basically say that the uncertainty set $\Omega$ is presented as a finite union of nonempty subsets. Condition C3) translated as one requiring the existence of a common quadratic Lyapunov function for any subset $\Omega_{i}$ further facilitates the process of decomposition. It is well known that such a finite-cover can be found under assumptions A1)-A3) (see, e.g., [1] and [5] for details). The computational complexity of decomposing the uncertainty set, in general, depends on many factors including the "size" of the set, its dimension and "stabilizability" properties, and has to be evaluated on a case by case basis. The key observation used in the localization technique is the following fact: Given any parameter vector $\theta \in \Omega_{j}$ and a control gain $K_{i(t)}$ for some $i(t), j=1, \cdots, L$. Suppose that $i(t)=j$, then it follows from (3) that

$$
\left|\theta_{j}^{T} \phi(t-1)-y(t)\right| \leq r_{j}\|\phi(t-1)\|+\bar{\xi}+\bar{\eta}(t-1) .
$$

If the above inequality is violated at any time instant, we know that the switching index $i(t)$ is wrong (i.e., $i(t) \neq j$ ), so it can be eliminated (falsified). The unique feature of the localization technique comes from the fact that violation of (4) allows us not only to eliminate a single index, $i(t)$ (if $i(t) \neq j$ ) from the set of possible controller indices, but many others. We now describe the localization algorithm. Let $I(t)$ denote the set of "admissible" control gain indices at time $t$ and initialize it to be $I\left(t_{0}\right)=\{1,2, \cdots, L\}$. Choose any initial switching index $i\left(t_{0}\right) \in I\left(t_{0}\right)$. For $t>t_{0}$, define $\hat{I}(t)=\{j:(4)$ holds, $j=1, \cdots, L\}$. Then, the localization algorithm is simply given by

$$
I(t)=I(t-1) \cap \hat{I}(t) \quad \forall t>t_{0} .
$$

The switching index is updated by taking ${ }^{1}$

$$
i(t)= \begin{cases}i(t-1), & \text { if } t>t_{0} \text { and } i(t-1) \in I(t) \\ \text { any member of } I(t), & \text { otherwise. }\end{cases}
$$

One possible way to view the localization technique is to interpret it as family set identification conducted on a finite set of elements.

Lemma 3.1 [9]: The localization algorithm given in (5) and (6) applied to an LTI plant (1) possesses the following properties: i) $I(t) \neq \emptyset$, $\forall t$ ii) There exists a switching index $j \in I(t)$ for all $t$ such that the closed-loop system with $u(t)=K_{j} x(t)$ is globally stable. The following theorem contains the main result for the LTI case.

Theorem 3.1: The localization algorithm given in (5) and (6) will guarantee the following properties when $\epsilon$ (i.e., the "size" of unmodeled dynamics) is sufficiently small.

i) The closed-loop system is globally $\bar{\xi}$-exponentially stable, i.e., there exists constants $M_{1}>0,0<\rho<1$, and a function $M_{2}(\cdot): \boldsymbol{R}_{+} \rightarrow \boldsymbol{R}_{+}$with $M_{2}(0)=0$ such that

$$
\|x(t)\| \leq M_{1} \rho^{\left(t-t_{0}\right)}\left\|x\left(t_{0}\right)\right\|+M_{2}(\bar{\xi})
$$

holds for all $t \geq t_{0}$ and $x\left(t_{0}\right)$.

ii) The switching sequence $\left\{i\left(t_{0}\right), i\left(t_{0}+1\right), \cdots\right\}$ is finitely convergent.

Proof: By Lemma 3.1 and the index update rule (6), the total number of switchings made by the controller is finite. Let $\left\{t_{1}, t_{2}, \cdots, t_{l}\right\}$ be a finite set of switching instants. By virtue of (1)-(4) the behavior of the closed-loop system between any two consecutive instants $t_{s}, t_{s+1}, 1 \leq s \leq l-1, t_{s+1} \geq t_{s}$ is described by

$$
\begin{aligned}
x(t+1) & =\left(A(\theta)+B(\theta) K_{i\left(t_{s}\right)}\right) x(t)+E(\xi(t)+\eta(t)) \\
& =\left(A\left(\theta_{i\left(t_{s}\right)}\right)+B\left(\theta_{i\left(t_{s}\right)}\right) K_{i\left(t_{s}\right)}\right) x(t)+E \psi(t) .
\end{aligned}
$$

Because no switching occurs between $t_{s}$ and $t_{s+1}$ we have $|\psi(t)| \leq$ $r_{i\left(t_{s}\right)}\|\phi(t)\|+\bar{\xi}+\bar{\eta}(t)$. We claim that due to the structure of the parameter dependent matrices $A(\theta)$ and $B(\theta)$, we can introduce fictitious parameters $\Delta \theta(t)$ and $\hat{\xi}(t)$ satisfying the relations

$$
\begin{gathered}
x(t+1)=\left(A\left(\theta_{i\left(t_{s}\right)}+\Delta \theta(t)\right)+B\left(\theta_{i\left(t_{s}\right)}+\Delta \theta(t)\right) K_{i\left(t_{s}\right)}\right) \\
\cdot x(t)+E \hat{\xi}(t)
\end{gathered}
$$

and $\Delta \theta(t):\|\Delta \theta(t)\| \leq r_{i\left(t_{s}\right)}$ and $|\hat{\xi}(t)| \leq \bar{\xi}+\bar{\eta}(t)$. Indeed, this follows from the fact that the last equation in (8) can be rewritten as $y(t+1)=\theta_{i\left(t_{s}\right)}^{T} \phi(t)+\psi(t)$ and that $\max _{\|\Delta \theta\| \leq 1}\left\|\Delta \theta^{T} \phi(t)\right\|=$ $\|\phi(t)\|$ holds for any $\phi(t)$. By Definition 3.1 and Condition C3) (9) with $\hat{\xi}(t) \equiv 0$ and $t_{s}$ being fixed is quadratically stable. This guarantees the existence of a matrix $H_{s}^{T}=H_{s}>0$ such that

$$
\begin{array}{r}
P_{s}=\max _{\|\Delta \theta(t)\| \leq r_{i\left(t_{s}\right)}} \| A\left(\theta_{i\left(t_{s}\right)}+\Delta \theta(t)\right)+ \\
\cdot\left(_{i\left(t_{s}\right)}+\Delta \theta(t)\right) \\
\cdot K_{i\left(t_{s}\right)} \|_{H_{s}}<1 .
\end{array}
$$

Here $\|x\|_{H}=\left(x^{T} H x\right)^{1 / 2}$ and for any $A \in \boldsymbol{R}^{n \times n},\|A\|_{H}=$ $\max _{\|x\|_{H}=1}\|A x\|_{H}$. Equation (9) along with the property of quadratic stability guarantee that between any two consecutive

${ }^{1}$ In fact, there are "clever" ways of selecting $i(t)$ when $i(t-1)$ is falsified (see [9] for details). 
switchings the closed-loop system behaves as an exponentially stable LTI system subject to small parametric perturbations $\Delta \theta(t)$ and bounded disturbance $\hat{\xi}(t)$. Further, this property holds regardless of the evolution of the plant parameters. This is the key point making the rest of the proof transparent. Let $\bar{\eta}(t) \equiv 0$, then it follows from (9) and (10) that

$$
\left\|x\left(t_{s}+1\right)\right\|_{H_{s}} \leq P_{s}\left\|x\left(t_{s}\right)\right\|_{H_{s}}+\hat{\xi}_{t_{s}}
$$

where $\hat{\xi}_{t_{s}}=\max _{|\xi|<\bar{\xi}}\|E \xi\|_{H_{s}}$.

Similarly

$$
\begin{aligned}
&\left\|x\left(t_{s}+2\right)\right\|_{H_{s}} \leq P_{s}^{2}\left\|x\left(t_{s}\right)\right\|_{H_{s}}+\left(P_{s}+1\right) \hat{\xi}_{t_{s}} \\
& \ldots \\
&\left\|x\left(t_{s}+k\right)\right\|_{H_{s}} \leq P_{s}^{k}\left\|x\left(t_{s}\right)\right\|_{H_{s}}+\hat{\xi} \sum_{i=1}^{k} P_{s}^{i-1} \\
&\left\|x\left(t_{s}+k\right)\right\| \leq\left(\lambda_{\max }\left(H_{s}\right) / \lambda_{\min }\left(H_{s}\right)\right)^{1 / 2} P_{s}^{k}\left\|x\left(t_{s}\right)\right\| \\
&+\hat{\xi}_{t_{s}} \sum_{i=1}^{k} P_{s}^{i-1} / \lambda_{\min }\left(H_{s}\right)^{1 / 2} .
\end{aligned}
$$

Denote

$$
\begin{aligned}
M & =\max _{1 \leq i \leq L}\left(\lambda_{\max }\left(H_{i}\right) / \lambda_{\min }\left(H_{i}\right)\right)^{1 / 2} \\
\rho & =\max _{1 \leq i \leq L} P_{i}<1 \\
M(\bar{\xi}) & =\max _{1 \leq i \leq L} \hat{\xi}_{i} /\left(\lambda_{\min }\left(H_{i}\right)\right)^{1 / 2} \sum_{j=1}^{\infty} P_{i}^{j-1}<\infty .
\end{aligned}
$$

Since $\theta_{i(t)} \in \theta\left(I_{t_{0}}\right), K_{i(t)} \in\left\{K_{i}\right\}_{i=1}^{L}$ for all $t \in \boldsymbol{N}, i(t) \in I_{t_{0}}$ there exist constants $0<M_{0}<\infty, \gamma_{0}=\max _{|\xi|<\bar{\xi}}\|E \xi\|<\infty$ such that $\left\|x\left(t_{s}\right)\right\| \leq M_{0}\left\|x\left(t_{s}-1\right)\right\|+\gamma_{0}$ for any switching instant $t_{1} \leq t_{s} \leq t_{l}$. Hence, combining the last inequality with (14)-(16) leads to

$$
\begin{aligned}
{\left[t_{0}, t_{l}\right): \quad\left\|x\left(t_{1}\right)\right\| } & \leq M_{0}\left\|x\left(t_{1}-1\right)\right\|+\gamma_{0} \\
& \leq M_{0} M \rho^{t_{1}-t_{0}-1}\left\|x\left(t_{0}\right)\right\|+M_{0} M(\bar{\xi})+\gamma_{0} \\
\left\|x\left(t_{2}\right)\right\| & \leq M_{0}\left\|x\left(t_{2}-1\right)\right\|+\gamma_{0} \\
& \leq M_{0}^{2} M^{2} \rho^{t_{2}-t_{0}-2}\left\|x\left(t_{0}\right)\right\|+\hat{M}_{2}(\bar{\xi})
\end{aligned}
$$

where

$$
\begin{aligned}
& \hat{M}_{2}(\bar{\xi})=M_{0}\left(M\left(M_{0} M(\bar{\xi})+\gamma_{0}\right)+M(\bar{\xi})\right)+\gamma_{0} ; \\
& \ldots \\
& {\left[t_{l}, \infty\right): \quad\|x(t)\| \leq M_{0}^{l} M^{l} \rho^{t-t_{0}-l}\left\|x\left(t_{0}\right)\right\|+\hat{M}_{l}(\bar{\xi}) . }
\end{aligned}
$$

Denoting $M_{1}=\left(M_{0} M / \rho\right)^{l}, M_{2}(\bar{\xi})=\hat{M}_{l}(\bar{\xi})<\infty$ we obtain (7). To conclude the proof we note that due to the term $\bar{\eta}(t)$ in the algorithm of localization (4)-(6) the process of localization cannot be disrupted by the presence of small unmodeled dynamics. In view of A5), (11)-(19) it is easy to show that for a sufficiently small $\epsilon$

$$
\begin{aligned}
{\left[t_{l}, \infty\right): \quad\|x(t)\| \leq } & M_{0}^{l} M^{l} \rho^{t-t_{0}-l}\left\|x\left(t_{0}\right)\right\| \\
& +\hat{M}_{l}(\bar{\xi})+M_{\eta} \epsilon\left\|x\left(t_{0}\right)\right\|
\end{aligned}
$$

with $M_{\eta}$ being a positive constant independent of $x\left(t_{0}\right)$. Therefore, $\|x(t)\| \leq\left(M_{1} \rho^{t-t_{0}}+M_{\eta} \epsilon\right)\left\|x\left(t_{0}\right)\right\|+\hat{M}_{l}(\bar{\xi})$ is valid for all $t_{0} \in \boldsymbol{N}$, $t \geq t_{l}$. From the last inequality and A5) exponential stability of the closed-loop system is easily established.

\section{LOCALIZATION TECHNIQUE FOR LTV PLANTS}

For the LTV plants, the general structure of the switching controller is similar to the LTI case except that the localization algorithm needs some modification. More specifically, at each $t>t_{0}$, the set $\hat{I}(t)$ is computed as above with $r_{j}$ being replaced by $\left(r_{j}+q\right)$, that is

$$
\begin{aligned}
& \hat{I}(t)=\left\{j: \mid \theta_{j}^{T} \phi(t-1)-\right. y(t) \mid \leq\left(r_{j}+q\right)\|\phi(t-1)\| \\
&+\bar{\xi}+\bar{\eta}(t-1), j=1, \cdots, L\} .
\end{aligned}
$$

By doing this we make the decomposition of the uncertainty set $\Omega$ slightly redundant. This avoids rapid switching of the controller caused by the parameters drifting slowly along the boundary of neighboring subsets. The localization set $I(t)$ is updated by

$$
I(t)= \begin{cases}I(t-1) \cap \hat{I}(t), & \text { if } I(t-1) \cap \hat{I}(t) \neq \emptyset \\ \hat{I}(t), & \text { otherwise. }\end{cases}
$$

Once (or if) the algorithm of localization has falsified all the indexes it simply disregards all the past measurements except for the most recent one and the process of localization continues. This forgetting scheme eliminates the need for persistency of excitation required in many adaptive control schemes.

Theorem 4.1: The localization scheme described above guarantees the following properties when $\epsilon$ (i.e., the "size" of unmodeled dynamics) is sufficiently small.

i) The closed-loop system is globally $\bar{\xi}$-exponentially stable if $M_{1}^{(1+[(N \alpha / q)]) l} \rho^{N}<1$ where $M_{1}$ and $\rho$ are constants in (7), $\alpha$, $N$ are constants used in Assumption A6), $q$ is given in Condition C3), and $l$ denotes the maximum number of switchings for the LTI plant.

ii) The switching sequence $\left\{i\left(t_{0}\right), i\left(t_{0}+1\right), \cdots\right\}$ is finitely convergent if the plant is LTI.

Proof: Relying on the proof of Theorem 3.1 it suffices to show that the sufficiently low "average frequency" of the controller switching implies global stability. Consider the behavior of the closed-loop system over $T=[t, t+\tau N]$ with $\tau, t \in N$ being arbitrary constants. Let $s$ denote the number of switchings made by the controller over $T$. Following the proof of Theorem 3.1 we establish that (provided that $\bar{\eta}(t) \equiv 0$ )

$$
\|x(t+\tau N)\| \leq\left(M_{0} M / \rho\right)^{s} \rho^{\tau N}\|x(t)\|+\hat{M}_{s}(\bar{\xi})
$$

$$
\lim _{\bar{\xi} \rightarrow 0} \hat{M}_{s}(\bar{\xi})=0, \quad 0<\rho<1 .
$$

We recall that $l$ denotes an upper bound on the total number of switchings made by the controller applied to the LTI plant (1). It is straightforward to verify that

$$
s \leq l \tau+1+(1+[\tau N /(q / \alpha)]) l=(1+[N \alpha / q]) l \tau+(l+1) .
$$

Therefore, (23) is transformed into

$$
\|x(t+\tau N)\| \leq\left(\bar{M}^{1+[N \alpha / q]) l} \rho^{N}\right)^{\tau} \bar{M}^{(l+1)}\|x(t)\|+\hat{M}_{s}(\bar{\xi})
$$


where $\bar{M}=M_{0} M / \rho$. Requiring $\left(\bar{M}^{(1+[N \alpha / q]) l} \rho^{N}\right)^{\tau} \bar{M}^{(l+1)}<1$ we have

$$
\bar{M}^{(1+[N \alpha / q]) l} \rho^{N} \leq \bar{M}^{-(l+1) / \tau} .
$$

Since the term $\bar{M}^{-(l+1) / \tau}$ is increasing in $\tau$ and $\lim _{\tau \rightarrow \infty} \bar{M}^{-(l+1) / \tau}=1$ the inequality $\bar{M}^{(1+[N \alpha / q]) l} \rho^{N}<1$ being true for a sufficiently large $N$ and a sufficiently small $\alpha$ guarantees the existence of a finite $\tau$ such that (27) holds. Hence, $\|x(t+\tau N)\| \leq \beta\|x(t)\|+\hat{M}_{s}(\bar{\xi})$ holds for some $0<\beta<1$ and any $t \in \boldsymbol{N}$. Now we need to show that the last inequality being true for all $t \in N$ implies exponential stability in the sense of Definition 2.1. Since

$$
\begin{aligned}
& \left\|x\left(t_{0}+k \tau N\right)\right\| \\
& \quad \leq \beta^{k}\left\|x\left(t_{0}\right)\right\|+\sum_{i=1}^{k} \beta^{i-1} \hat{M}_{s}(\bar{\xi}) ; \quad \forall k \in N
\end{aligned}
$$

$$
\begin{aligned}
& \left\|x\left(t_{0}+k \tau N+i\right)\right\| \\
& \leq G_{1}\left\|x\left(t_{0}+k \tau N\right)\right\|+G_{2} ; \quad \forall i \in[1, N-1] \\
& \quad \forall k \in N
\end{aligned}
$$

for some positive constants $G_{1}, G_{2}$, and $\sum_{i=1}^{k} \beta^{i-1}<\infty ; \forall k \in N$ the exponential stability is proved in a straightforward way. Using the same arguments as in the proof of Theorem 3.1 the result above is easily extended to the case $\bar{\eta}(t) \neq 0$.

Remark 4.1: The condition $M_{1}^{(1+[(N \alpha / q)]) l} \rho^{N}<1$ in i) of Theorem 4.1 automatically holds for LTI plants because $N \rightarrow \infty$ and $\alpha=0$ in this case.

\section{Simulation EXAMPLE}

Consider the following family of unstable and pointwise nonminimumphase LTV plants:

$$
\begin{aligned}
y(t)=1.2 y(t-1)-1.22 y(t-2) & +b_{1}(t) u(t-1) \\
& +b_{2}(t) u(t-2)+\xi(t)
\end{aligned}
$$

with the exogenous disturbance $\xi(t)$ being uniformly distributed on the interval $[-0.1,0.1]$. The a priori uncertainty bounds are given by $b_{1}(t) \in[-1.6,-0.15] \cup[0.15,1.6], b_{2}(t) \in[-2,-1] \cup[1,2]$. The results of localization on the finite set $\left\{\theta_{i}\right\}_{i=1}^{600}$ are presented in Fig. 1(a)-(e). A pole placement technique was used to compute the gains $\left\{K_{i}\right\}_{i=1}^{600}$ with the poles of the nominal closed-loop system being $(0,0.07,0.1)$. The interesting features of the localization technique observed in simulations include a low sensitivity of the speed of localization to the total number of fixed controllers and the switching index update rule.

\section{CONCLUSION}

The switching adaptive control approach presented in this paper is based on a localization method which is conceptually different from the existing switching adaptive schemes. The unique feature of localization based switching adaptive control is its rapid falsification capabilities. For further details and more advanced applications of the localization method, see [9].
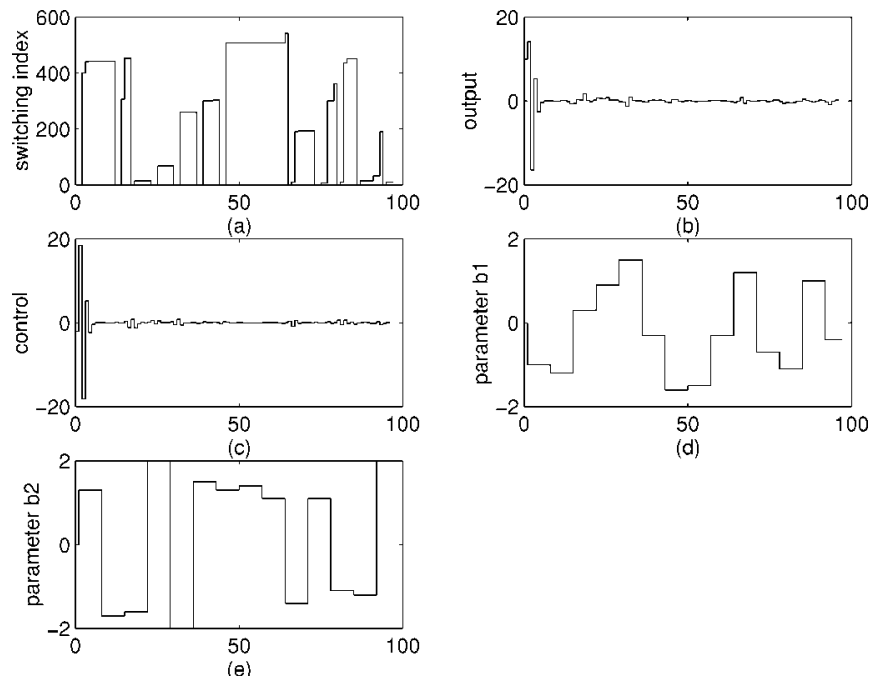

Fig. 1. Example of localization: Parameters jump every seven steps.

\section{REFERENCES}

[1] M. Fu and B. R. Barmish, "Adaptive stabilization of linear systems via switching control," IEEE Trans. Automat. Contr., vol. 31, pp. 1097-1103, Dec. 1986.

[2] G. Kreisselmeier, "Adaptive control of a class of slowly time-varying plants,” Syst. Contr. Lett., vol. 8, pp. 97-103, 1986.

[3] B. Martensson, "The order of any stabilizing regulator is sufficient $a$ priori information for adaptive stabilizing," Syst. Contr. Lett., vol. 6, no. 2, pp. 87-91, 1985.

[4] R. H. Middleton and G. C. Goodwin, "Adaptive control of time-varying linear systems," IEEE Trans. Automat. Contr., vol. 33, pp. 150-155, Jun. 1988.

[5] A. S. Morse, "Supervisory control of families of linear set-point controllers," in Proc. 32nd CDC, Dec. 1993.

[6] K. S. Narendra and J. Balakrishnan, "Intelligent control using fixed and adaptive models," in Proc. 33rd CDC, Dec. 1994.

[7] R. D. Nussbaum, "Some remarks on a conjecture in parameter adaptive control," Syst. Contr. Lett., vol. 3, pp. 243-246, Nov. 1983.

[8] K. S. Tsakalis and P. A. Ioannou, "Adaptive control of LTV plants: A new model reference controller structure," IEEE Trans. Automat. Contr., vol. 34, pp. 1038-1046, Oct. 1989.

[9] P. V. Zhivoglyadov, R. H. Middleton, and M. Fu, "Localization based switching adaptive control for time varying discrete time systems," Dept. Elect. and Comp. Eng., Univ. of Newcastle, Australia, Tech. Rep. N 9709, 1997. 